\title{
Optimal level of purple acid phosphatase 5 is required for maintaining complete resistance to Pseudomonas syringae
}

\section{OPEN ACCESS}

Edited by:

Etienne Yergeau,

National Research Council Canada

Canada

Reviewed by:

William Underwood,

University of California, USA

Raffaella Balestrini,

Consiglio Nazionale delle Ricerche,

Italy

${ }^{*}$ Correspondence:

Balakrishnan Prithiviraj,

Department of Environmental

Sciences, Faculty of Agriculture, Dalhousie University, 50 Pictou Road,

Truro, NS B2N 5E3, Canada bprithiviraj@dal.ca

${ }^{\dagger}$ Present Address:

Sridhar Ravichandran,

Eastern Cereal and Oilseed Research

Centre, Agriculture and Agri-Food Canada, Ottawa, Canada

Specialty section

This article was submitted to Plant Biotic Interactions,

a section of the journal Frontiers in Plant Science

Received: 06 May 2015

Accepted: 09 July 2015

Published: 04 August 2015

Citation:

Ravichandran S, Stone SL, Benkel B, Zhang J, Berrue $F$ and Prithiviraj $B$ (2015) Optimal level of purple acid phosphatase5 is required for maintaining complete resistance to

Pseudomonas syringae

Front. Plant Sci. 6:568.

doi: 10.3389/fpls.2015.00568

\section{Sridhar Ravichandran ${ }^{1 \dagger}$, Sophia L. Stone ${ }^{2}$, Bernhard Benkel ${ }^{3}$, Junzeng Zhang ${ }^{4}$, Fabrice Berrue ${ }^{5}$ and Balakrishnan Prithiviraj ${ }^{1 *}$}

${ }^{1}$ Department of Environmental Sciences, Faculty of Agriculture, Dalhousie University, Truro, NS, Canada, ${ }^{2}$ Department of Biology, Dalhousie University, Halifax, NS, Canada, ${ }^{3}$ Department of Plant and Animal Sciences, Faculty of Agriculture, Dalhousie University, Truro, NS, Canada, ${ }^{4}$ Aquatic and Crop Resource Development, National Research Council Canada, Halifax, NS, Canada, ${ }^{5}$ Department of Chemistry, University of Prince Edward Island, Charlottetown, PE, Canada

Plants possess an exceedingly complex innate immune system to defend against most pathogens. However, a relative proportion of the pathogens overcome host's innate immunity and impair plant growth and productivity. We previously showed that mutation in purple acid phosphatase (PAP5) lead to enhanced susceptibility of Arabidopsis to the bacterial pathogen Pseudomonas syringae pv. tomato DC3000 (Pst DC3000). Here, we report that an optimal level of PAP5 is crucial for mounting complete basal resistance. Overexpression of PAP5 impaired ICS1, PR1 expression and salicylic acid (SA) accumulation similar to pap5 knockout mutant plants. Moreover, plant overexpressing PAP5 was impaired in $\mathrm{H}_{2} \mathrm{O}_{2}$ accumulation in response to Pst DC3000. PAP5 is localized in to peroxisomes, a known site of generation of reactive oxygen species for activation of defense responses. Taken together, our results demonstrate that optimal levels of PAP5 is required for mounting resistance against Pst DC3000 as both knockout and overexpression of PAP5 lead to compromised basal resistance.

Keywords: purple acid phosphatase5 (PAP5), Pseudomonas syringae pv. tomato DC3000, disease resistance, Arabidopsis, reactive oxygen species (ROS)

\section{Introduction}

Plants are constantly exposed to a diverse array of microbial pathogens. In nature, plants have evolved mechanism(s) to restrict most pathogens (non-host disease resistance) and also reduce pathogen ingress (basal resistance or PAMP-triggered immunity) (Bittel and Robatzek, 2007). Activation of defense response begins with the recognition of conserved molecular signatures or PAMP (Pathogen-Associated Molecular Pattern) by pattern recognition receptors (PRRs) localized on the plasma membrane and in the cytoplasm (Chisholm et al., 2006; Jones and Dangl, 2006). PAMPs are microbial molecular signatures (e.g., flagellin, bacterial lipopolysaccharides, elongation factor, chitin, and $\beta$-glucan) that are absent in the host (Boller and Felix, 2009; Schwessinger and Ronald, 2012). Following PAMP perception, the PRRs initiate complex signaling networks that are associated with rapid synthesis of reactive oxygen species, activation MAP kinase signaling and pathogenesis related (PR) genes leading to PAMP triggered immunity (PTI) (Chisholm et al., 2006). However, well-adapted microbial pathogens have evolved the means to subvert defense signaling and breach PTI. 
Plants are exposed to pathogens that have diverse infection strategies and therefore activation of appropriate, pathogen specific defense responses is vital for plant growth and productivity (Glazebrook, 2005). Structural alteration in cell wall including waxy cuticle layer, deposition of callose, suberin, and lignifications of cell wall provide protection contributing to non-host disease resistance (Dangl and Jones, 2001). Upon pathogen recognition, immediate early response genes including glutathione S transferase 6 (GST) and immediate early induced glucosyltransferase (IEGT) detoxify and protect cells from oxidative stress (Uquillas et al., 2004 and references therein). Salicylic acid (SA) dependent defense marker gene such as PR1 is induced later during pathogenesis involving the key signal transducer NPR1 (non-expressor of pathogenesis related genes 1) (Schenk et al., 2000). Specific response to pathogens is also mediated by gene-for-gene recognition leading to the activation of resistance (R) genes or recently termed effector-triggered immunity (ETI) in host (Nimchuk et al., 2003; Chisholm et al., 2006). Activation of $\mathrm{R}$ gene is usually accompanied by production of reactive oxygen species (ROS) leading to hypersensitive responses (HR) to restrict the spread of pathogen (Glazebrook, 2005). The localized cell death triggers systemic acquired resistance (SAR) to confer resistance throughout the plant (Baker et al., 1997). Hypersensitive response is also associated with induction of diverse group of defense related and pathogenesis related (PR) genes.

Plants defense responses are primarily associated with salicylic acid (SA), jasmonic acid (JA), and ethylene (ET) (Vlot et al., 2009). SA regulates the activation of defense responses against most biotrophic, hemi-biotrophic pathogens (Durner et al., 1997) and also mediates the establishment of systemic acquired resistance (SAR) (Grant and Lamb, 2006). By contrast, JA and ET operate synergistically to confer resistance against necrotrophic pathogens and herbivorous insects. JA is also associated with induced systemic resistance (ISR) elicited by rhizobacterial strains that promote plant growth and enhances resistance to various bacterial and fungal pathogens (Ton et al., 2002). In recent years, the role of other phytohormones including abscisic acid (ABA), auxins, gibberellins (GA), cytokines (CK), and brassinosteriods (BR) have started to emerge (Mutka et al., 2013) reviewed by Bari and Jones (2009). Apart from plant hormones, class of secondary metabolites including phenyl proponoid, glucosinolates, terpenoids, and phytoalexins aid in the protection of plant against most biotic stress (Kliebenstein, 2004). Synthesis and secretion of anti-microbial compounds confer selective advantage to curb pathogen invasion. Over 100,000 low-molecular-mass compounds derived from isoprenoid, polypropanoid, polyketide pathways are known to enhance defense against microbial infections (Dixon, 2001).

Purple acid phosphatases (PAPs) belong to a family of binuclear metalloenzymes and have been identified and characterized in numerous plants, animals, and a limited number of microorganisms (Mitic et al., 2006; Schenk et al., 2008). All PAPs contain dinuclear metal ions and a characteristic set of seven invariant residues, which coordinate the metal ions within the active site (reviewed by Mitic et al., 2006; Schenk et al., 2008). PAPs have been implicated in an array of biological functions. Most PAPs have been classified as non-specific acid phosphatases that catalyze the hydrolysis of inorganic phosphate $(\mathrm{Pi})$ from various monoesters and anhydrides substrates (Olczak and Watorek, 2003). The physiological role of plant PAPs is predominantly associated with the regulation of Pi uptake and recycling (Li et al., 2002; Veljanovski et al., 2006). However, previous studies have also revealed roles for plant PAPs in other biological functions, including peroxidation (Del Pozo et al., 1999), ascorbate recycling (Zhang et al., 2008), mediation of salt tolerance (Liao et al., 2003), and regulation of cell wall carbohydrate biosynthesis (Kaida et al., 2009).

Recently, we demonstrated that the loss of purple acid phosphatase5 (PAP5) in Arabidopsis leads to enhanced susceptibility to virulent Pseudomonas syringae pv. tomato DC3000 (Pst DC3000). Arabidopsis plants carrying a mutation in PAP5 exhibited a defect in the expression of pathogenesis related (PR) genes including Pathogenesis Related gene 1 (PR1), Isochorismate synthase1 (ICS1) and plant defensin1.2 (PDF1.2). This study also revealed that pap5 plants failed to accumulate $\mathrm{H}_{2} \mathrm{O}_{2}$ in response to Pst DC3000 infection compared to wild-type plants (Ravichandran et al., 2013). One of the earliest responses to pathogen infection is generation of reactive oxygen intermediates ROIs $\left(\mathrm{O}_{2}^{-}\right.$and $\left.\mathrm{H}_{2} \mathrm{O}_{2}\right)$. Although, ROIs such as hydroxyl radical (.OH), superoxide radical $\left(\mathrm{O}_{2}^{-}\right)$and hydrogen peroxide $\left(\mathrm{H}_{2} \mathrm{O}_{2}\right)$ are produced under normal metabolic processes. The rapid accumulation of ROS (also known as oxidative burst) cause oxidative cross linking of cell wall, activation of cellular signaling (protein phosphorylation) and induction of pathogenesis related (PR) genes (Alvarez et al., 1998). It is widely assumed that ROS production after pathogen recognition is associated with membrane bound $\mathrm{NADPH}$ oxidase in the apoplast (Lamb and Dixon, 1997). $\mathrm{H}_{2} \mathrm{O}_{2}$ generated in response to pathogen recognition induces salicylic acid (SA) and PR protein accumulation (Chamnongpol et al., 1998). A number of studies have indicated that ROS produced in response to pathogen recognition is located in the apoplast (reviewed by Torres et al., 2002). It is also evident that plants can produce ROS in other inter-cellular organelles including chloroplast, mitochondria and peroxisomes. However, the cellular homeostasis and concentration of ROS is highly regulated by enzymes such catalase, peroxidase and superoxide dismutase (Foyer and Noctor, 2003).

Having demonstrated that the loss of PAP5 impaired plants innate immune responses (Ravichandran et al., 2013), we wanted to determine if overexpression of PAP5 results in enhanced disease resistance. Further, in silico predictions failed to detect signal peptides on $P A P 5$, hence we wanted to experimentally verify the prediction by tagging PAP5 with a fluorescent label. Here, we report that the level of PAP5 is crucial for mounting complete resistance against Pst DC3000. Optimal levels of PAP5 are required for induction of $\mathrm{PR}$ genes and $\mathrm{SA}$ accumulation. Further, PAP5 was found to be peroxisomal localized and aid the generation of reactive oxygen species for activation of defense responses. 


\section{Results}

\section{Optimal Level of PAP5 Is Required for Complete Resistance to Pst DC3000}

Previously, we reported that loss of PAP5 resulted in enhanced susceptibility of Arabidopsis to the virulent Pst DC3000 (Ravichandran et al., 2013). Having demonstrated that the loss of PAP5 lead to enhanced susceptibility, we tested if overexpression of PAP5 will result in enhanced resistance. We generated transgenic plants overexpressing PAP5 under the control of constitutive cauliflower mosaic virus (CaMV) $35 \mathrm{~S}$ promoter. Plants exhibiting Basta resistance were chosen and plants were picked randomly to verify the abundance of PAP5 transcripts. Among the transgenic lines tested, two independent overexpressor lines 35S:PAP5-A and 35S:PAP5-B were chosen for further studies. Overexpressor lines 35S:PAP5-A and 35S:PAP5-B showed $\sim 8$ and 62 -fold increase in PAP5 transcripts respectively compared to wild-type (Col-0) plants (Figure 1A). There was no difference in the growth and development of both the overexpressing lines compared to wild-type plants.

For pathogenicity assay, plants were sprayed with suspension of Pst DC3000 as described in the methods. Interestingly, the overexpressor lines exhibited extensive chlorosis and increased susceptibility to Pst DC3000 compared to wild-type plants. Assessment of Pst DC3000 growth in plant apoplast revealed that both overexpressor lines had higher bacterial titers compared to wild-type plants (Figure 1B). Both overexpressor lines exhibited comparable levels of chlorosis and bacterial titers suggesting that the enhanced susceptibility is not due to the positional effects on insertion of the transgene. Although the overexpressor line 35S:PAP5-B constitutively expressed higher levels ( $\sim 62$ fold) of PAP5 compared to 35S:PAP5-A plants (Figure 1A) we did not observe any significant difference in susceptibility to Pst DC3000 (Figure 1B).

\section{Overexpression of PAP5 Impairs Pathogenesis Related (PR) Gene Expression and Alters $\mathrm{H}_{2} \mathrm{O}_{2}$ and Salicylic Acid Accumulation}

In contrast to our expectation, overexpression of PAP5 did not result in enhanced resistance. Therefore, we tested if expression of defense related genes are impaired in the overexpressor lines (35:PAP5-A and 35S:PAP5-B). The overexpressor lines and wild-type plants were spray inoculated with suspension of Pst DC3000 $\left(10^{8}\right.$ c.f.u $\left.\mathrm{ml}^{-l}\right)$ and leaf tissues were harvested for gene expression analysis. The expression of pathogenesis related protein gene1 (PR1), a commonly used marker gene associated with Pst DC3000 infection and SA-mediated defense responses was observed. There was no significant difference in the transcript abundance of $P R 1$ between the mock infected overexpressor lines and the wild-type plants. However, $P R 1$ was strongly induced in infected wild-type plants 24 h.p.i., whereas it was not induced in $\mathrm{OE}$ lines (Figure 2A). Although the expression of $P R 1$ was slightly higher in both overexpression lines at 48 h.p.i., the levels of $P R 1$ was significantly lower compared to infected wild-type plants (Figure 2A). We also tested the expression of isocorismate synthase1 (ICS1), which is responsible for $\sim 90 \%$ of pathogen induced SA production (Wildermuth

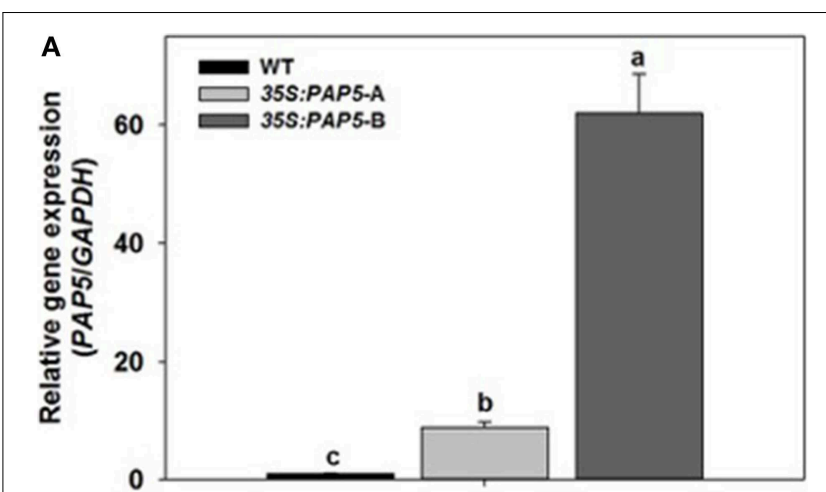

B

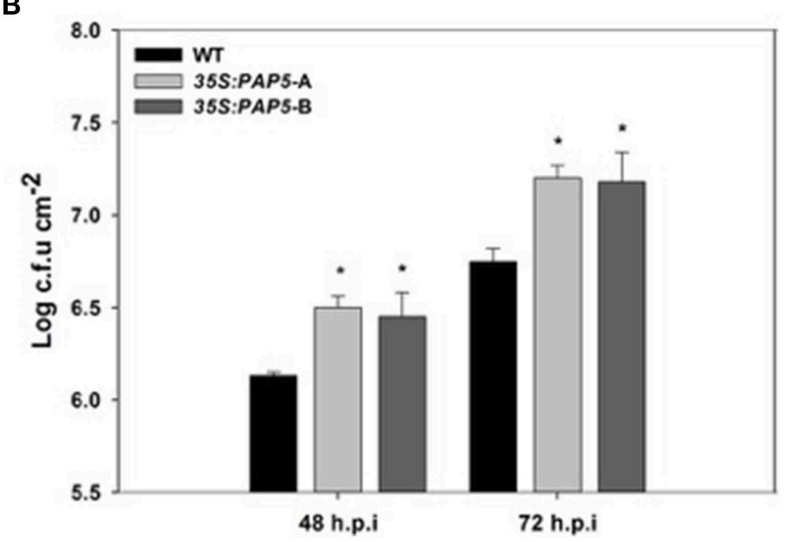

FIGURE 1 | Transgenic plants overexpressing PAP5 exhibit enhanced disease susceptibility. (A) Transcript levels of PAP5 in transgenic plants. Total RNA was extracted from wild-type and transgenic plants as described in materials and methods. Transcript levels of PAP5 was normalized to the expression of GAPDH in the same samples and expressed relative to the normalized transcript levels of wild-type plants. The bars represent the mean and standard deviation from two independent experiments. Significant differences $(P<0.05)$ are indicated by different letters. (B) Growth of Pst DC3000 in wild type and transgenic plants. Plants were inoculated with Pst DC3000 (10-8 c.f.u $\mathrm{ml}^{-1}$ ) and bacterial growth in plant apoplast was determined as described in the materials and methods. The bars represent the mean and standard deviation from values of three separate trials with six to eight replicates each trial. An asterisk indicates a significance increase in Pst DC3000 growth compared to wild-type plants (Student's $t$-test; $P<0.05$ ).

et al., 2002). As shown in Figure 2B, accumulation of ICS1 was $\sim 4$ fold higher in infected wild-type plants, whereas the expression of ICS1 was strongly suppressed in both the OE1, OE2 overexpressor lines. However, the expression of ICS1 in infected wild-type plants was similar to mock infected wild-type plants at 48 h.p.i. Moreover, the expression of ICS1 correlated with the expression of PR1.

To determine whether the decrease in the expression of ICS1 altered the concentration of salicylic acid (SA), we quantified the SA in the infected leaf tissue. We quantified SA in wild-type, overexpressor lines (35:PAP5-A and 35S:PAP5-B) and knockout line pap5-1 following Pst DC3000 infection. The concentration of SA increased in all Pst DC3000 infected plants. However, the SA levels in both the overexpressor lines and pap5-1 plants were only $\sim 60 \%$ of the wild-type plants (Figure 3A). In 


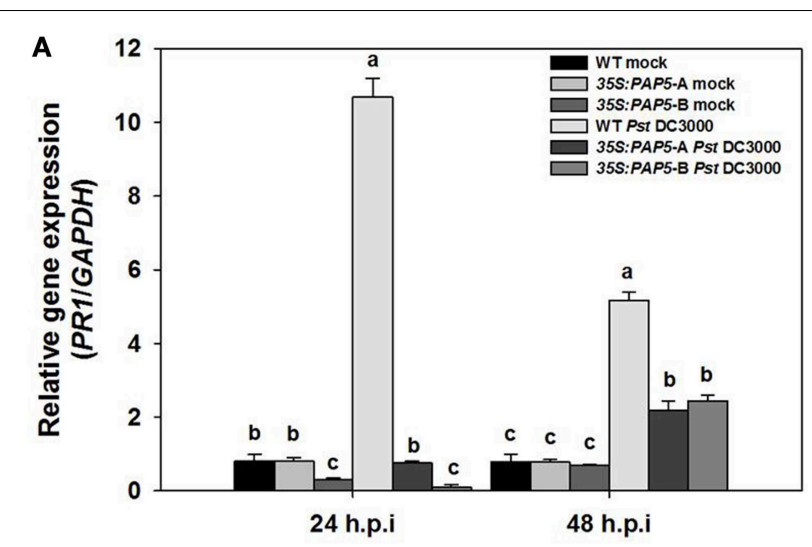

B

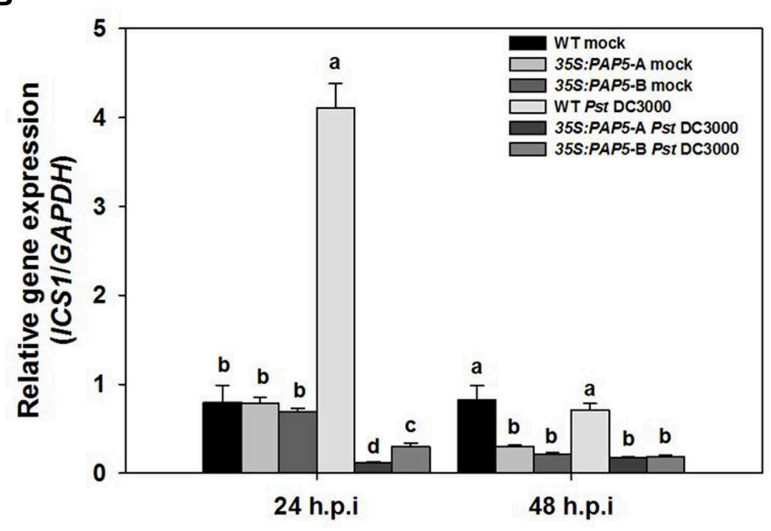

FIGURE 2 | Expression of ICS1 and PR1 is impaired in transgenic plants (35S:PAP5-A and 35S:PAP5-B). Plants were spray inoculated with Pst DC3000 (10 ${ }^{8}$ c.f.u $\mathrm{ml}^{-1}$ ) and RNA was extracted from leaf tissues sampled 24 and 48 h.p.i. Transcript levels were normalized to the expression of GAPDH in the same samples. The transcript levels were expressed relative to the normalized transcript levels of mock infected wild-type plants. The bars represent the mean and standard deviation from two independent experiments. Transcript levels of ICS1 (A) and PR1 (B) in mock and Pst DC3000 infected plants. Significant differences $(P<0.05)$ are indicated by different letters (Student's $t$-test; $P<0.05$ ).

addition, accumulation of hydrogen peroxide $\left(\mathrm{H}_{2} \mathrm{O}_{2}\right)$ generated in response Pst DC3000 was suppressed in both overexpressor lines (Figure 3B). These results suggest that the over expression of PAP5 impaired the transcription of ICS1, PR1, and SA accumulation subsequently similar to that of the loss-of-function pap5 mutant. Taken together, it is evident that optimal level of PAP5 is required for expression of ICS1 and PR1 and for accumulation of $\mathrm{H}_{2} \mathrm{O}_{2}$ and SA after Pst DC3000 infection. Further, these results suggest that the enhanced growth of Pst DC3000 in 35S:PAP5-A and 35S:PAP5-B is dependent on SA accumulation and SA-mediated defense responses.

\section{Sub-cellular Localization of PAP5}

To identify the sub-cellular localization of PAP5, a comprehensive in silico prediction was performed. Most in silico prediction revealed that PAP5 could be localized to the nucleus, cytosol, extracellular, endoplasmic reticulum, golgi bodies and to the extracellular space (http://suba.plantenergy. uwa.edu.au/). However, a search on http://www.cbs.dtu.dk/

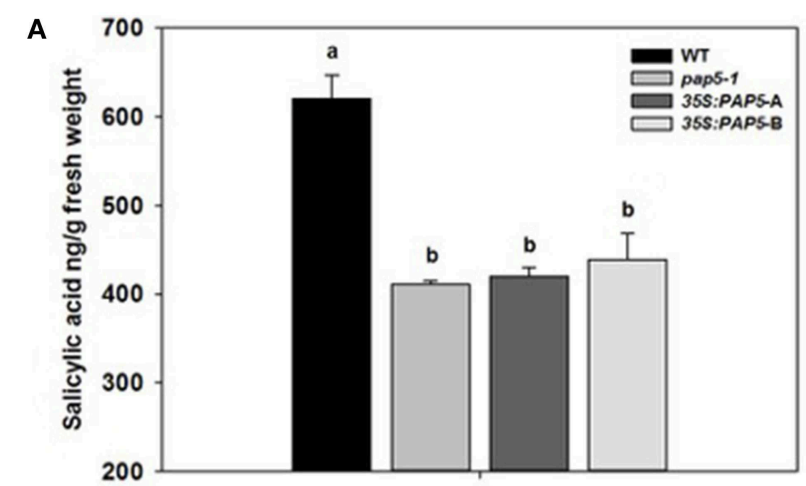

B

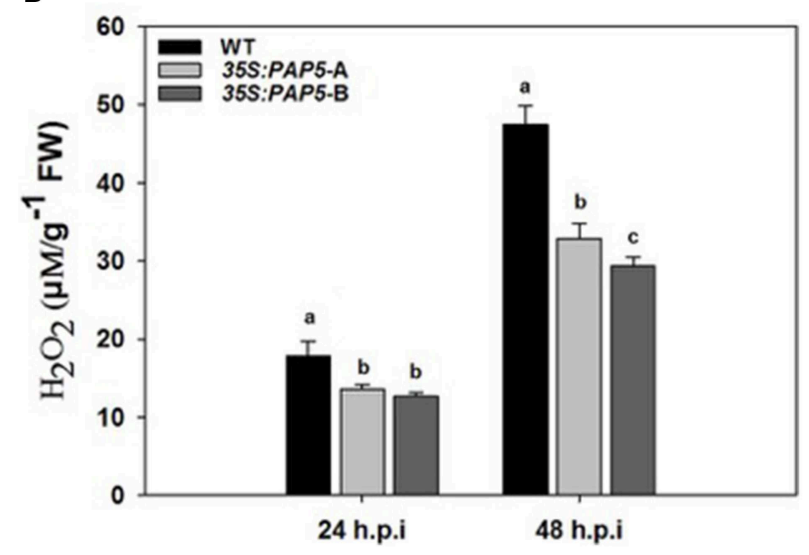

FIGURE 3 | Both loss and overexpression of PAP5 affect salicylic acid (SA) and $\mathrm{H}_{2} \mathrm{O}_{2}$ accumulation in Pst DC3000 infected plants. (A) Quantification of SA in pap5-1 and 35S:PAP5 plants following Pst DC3000 infection. Plants were spray inoculated with Pst DC3000 $\left(10^{8}\right.$ c.f.u ml $\left.{ }^{-l}\right)$ and leaf tissues were excised 48 h.p.i. The bars represent the mean and standard deviation from three replicates. Significant differences $(P<0.05)$ are indicated by different letters (Student's t-test; $P<0.05$ ). (B) Quantification of $\mathrm{H}_{2} \mathrm{O}_{2}$ following Pst DC3000 infection. The bars represent mean and $\mathrm{SD}$ of $\mathrm{H}_{2} \mathrm{O}_{2}$ accumulation. A significant difference $(P<0.05)$ in $\mathrm{H}_{2} \mathrm{O}_{2}$ production are indicated by different letters (Student's t-test; $P<0.05$ ).

services/SignalP/ revealed that PAP5 does not carry a signal peptide. To verify this contradictory in silico prediction, the coding region of PAP5 was fused to the YFP reporter gene under the control of CaMV 35S promoter. Confocal microscopy reveled YFP-PAP5 as rapidly moving punctate structures within the cytoplasm and faintly in the nucleus (Figure 4). To identify the cellular compartment, the agrobacterium strains carrying organelle specific markers (Nelson et al., 2007) were coinfiltered with YFP-PAP5 and leaf tissues were harvested at different time points for confocal microscopy. As shown in Figure 4, YFP-PAP5 showed a strong colocalization with peroxisomal specific marker (peroxisomal targeting signal 1; PTS1-CFP). Agrobacterium strains carrying YFP-PAP5 was also cofiltered with golgi (GmMan1 cytoplasmic tail and transmembrane) and plastid (rubisco targeting sequence) specific markers. In contrast to in silico predictions, YFP-PAP5 did not localize to golgi or plastid organelle specific markers (Figure S1).

Since the in silico prediction showed that PAP5 lack signal peptides, we wanted to determine if removal of specific stretch 


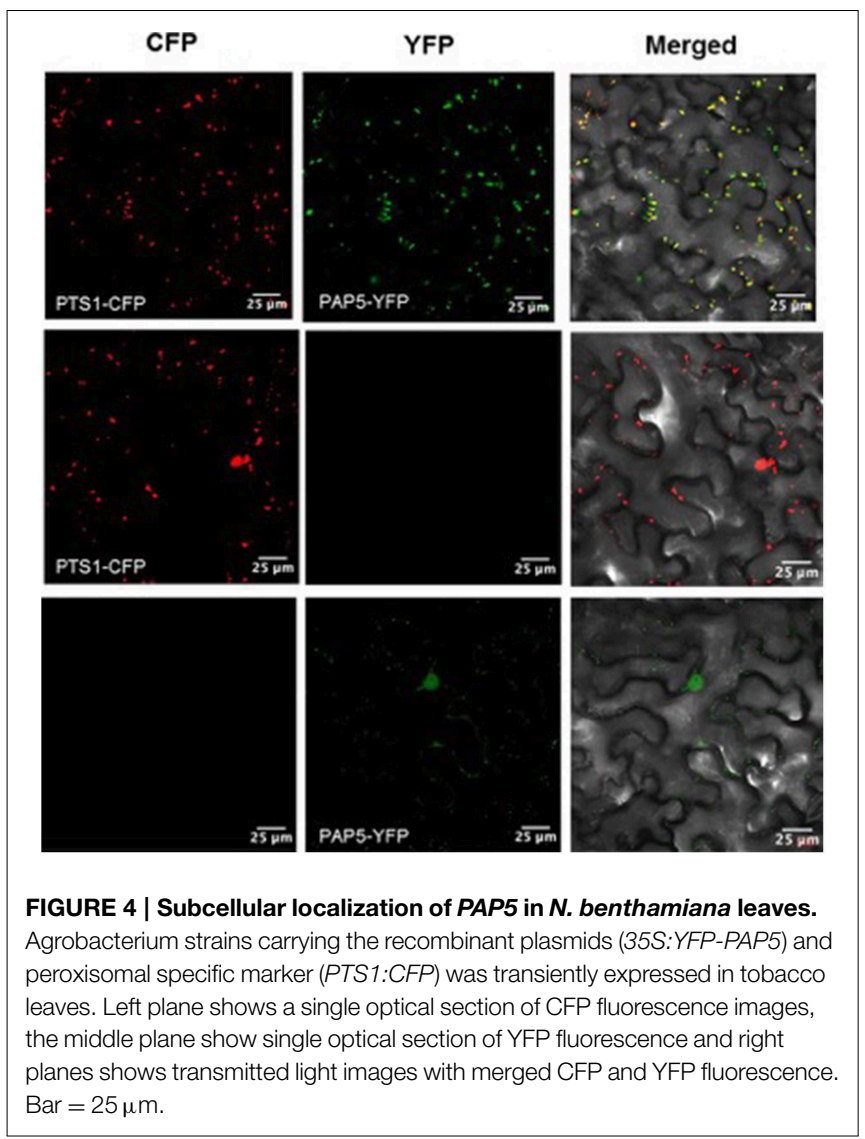

of amino acids from $\mathrm{C}$ - or $\mathrm{N}$-terminals affect the peroxisomal localization of PAP5. PAP5 constructs lacking a stretch of $\mathrm{N}$ terminal amino acids (MSLETFPPPA), referred as YFP:+30PAP5 failed to localize to peroxisomes (Figure 5). However, removal of amino acids RYYLPEEETI from the C-terminal (referred as YFP: -30PAP5) of PAP5 did not prevent the localization of PAP5 to peroxisomes. These results suggest that $\mathrm{N}$-terminal amino acids MSLETFPPPA are required for subcellular localization of PAP5.

\section{Discussion}

In this study, we showed that an optimal level of PAP5 is critical for mounting appropriate defense responses. Previous studies have revealed several molecular cues that regulate plant defense responses. Often, genes identified as positive regulator of defense responses are overexpressed to generate disease tolerant crops (Zhang et al., 2014). Interestingly, in some instances, both reduced and overexpression of a gene could result in the same phenotype. For example, OXI1 (Oxidative Signal-Inducible1), encoding a serine/threonine kinase has been shown to be required for complete activation of mitogen-activated protein kinase (MAPKs). oxi-1 null mutants showed enhanced susceptibility to virulent Hyaloperonospora arabidopsidis (formerly Paranospora parasitica) (Menke et al., 2004). Interestingly, transgenic plants overexpressing OXI1 (35S::OXI1) displayed enhanced susceptibility to both virulent Hyaloperonospora arabidopsidis and Pst DC3000 (Petersen et al.,

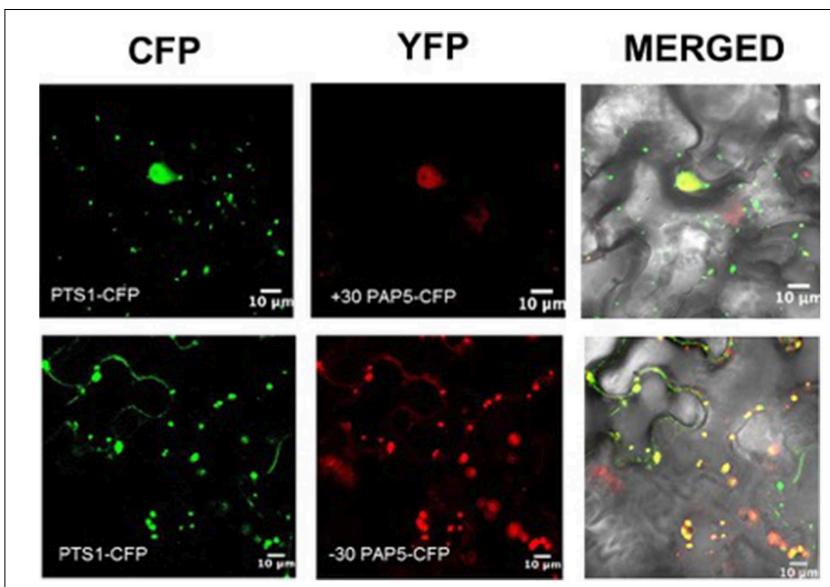

FIGURE 5 | Subcellular localization of PAP5 in $\boldsymbol{N}$. benthamiana leaves. Agrobacterium strains carrying the recombinant plasmids (+30PAP5:YFP or +30PAP5:YFP) and peroxisomal specific marker (PTS1-CFP) was transiently expressed in tobacco leaves. Left plane shows a single optical section of CFP fluorescence images, middle planes show a single optical section of YFP fluorescence and right planes shows transmitted light images with merged CFP and YFP fluorescence. +30PAP5:YFP lacks a stretch of N-terminal amino acids MSLETFPPPA. -30PAP5:YFP lack N-terminal amino acids MSLETFPPPA. Bar $=10 \mu \mathrm{m}$.

2009). Since both reduced and overexpression of PAP5 lead to enhanced susceptibility to Pst DC3000, we hypothesize that PAP5 could exist in complex with other proteins. Thus, constitutive expression of PAP5 (35S:PAP5) alone may not be sufficient for complete resistance. It is also possible that the prolonged expression of $P A P 5$ could negatively affect basal resistance against Pst DC3000. Previously, we identified that PAP5 is not expressed under normal growth conditions and are selectively induced only during prolonged Pi starvation and early stage of Pst DC3000 (6 h post inoculation) (Ravichandran et al., 2013). Hence, constitutive overexpression of PAP5 is not optimal and may perturb and impair its function following Pst DC3000. Previous studies have also shown that both overexpression and partial loss of FIP1 [FIN (Far-red insensitive) 219 Interacting Protein] resulted in hyposensitive hypocotyl phenotype under continuous Far Red (FR) light (Chen et al., 2007). FIP1 was also shown to exhibit glutathione S-transferse activity which lead to delayed flowering phenotype under long-day conditions. Similarly, loss and overexpression of EBS (Early Bolting in Short Days) showed early flowering, a dwarf phenotype and reduced fertility (Pineiro et al., 2003). EBS encodes a nuclear protein with homeodomain $\mathrm{Zn}$ finger that regulate chromatin remodeling and repress the initiation of flowering in short days.

Pathogen recognition triggers generation of reactive oxygen intermediates (ROIs), which is required for activation of defense responses (Torres et al., 2002). It is also evident that the generation of ROI occurs within hours of pathogen infection (Alvarez et al., 1998). Since PAP5 is induced only during the earlier stages (6h.p.i) of Pst DC3000 infection and the localization of PAP5 in peroxisome (Figure 4) suggests that PAP5 may act as a component of ROI generation. Hence we hypothesized that PAP5 might exist in complex with other 
proteins, a comprehensive in silico prediction was performed to identify proteins that may potentially interact with PAP5. Most in silico prediction searches on Bio-Analytic Resource for Plant Biology (BAR), Biological General Repository for Interaction Datasets (BioGRID) and GeneMANIA failed to detect any physical interaction. Moreover, most subcellular tools including SUBA, TargetP, and WoLF PSORT failed to identify the peroxisomal targeting sequences of PAP5. Previous studies have also shown that most in silico prediction programs fail to identify signature peroxisomal targeting sequences (Nelson et al., 2007). Few in silico prediction showed that PAP5 is targeted to the extracellular space (http://suba.plantenergy.uwa.edu.au/; http:// wolfpsort.org/).

In mammals, the high expression of PAPs in macrophages and increased ROS production that mediates microbial killing has been demonstrated (Kaija et al., 2002). Although ROS are produced under normal metabolic processes, the role of ROS in signaling is largely dependent on the rate of synthesis and is controlled by antioxidative enzymes such as catalase and peoxidases in peroxisomes (Nyathi and Baker, 2006). Previously, catalase deficient plants have been shown to display marked perturbation of intracellular redox and cellular homeostasis (Vandenabeele et al., 2004). Such perturbation is associated with accumulation of salicylic acid (SA) and induction of pathogenesis related (PR) genes (Chamnongpol et al., 1998). Similarly, catalase deficient Arabidopsis (cat2) plants showed increased peroxisomal $\mathrm{H}_{2} \mathrm{O}_{2}$ (Chaouch et al., 2010). Peroxisomal $\beta$-oxidation is also attributed to the degradation of various straight and branched chain fatty acids (Baker et al., 1997). Derivatives of $\beta$-oxidation such as cyclic oxylipins also play a significant role in the synthesis of plant hormones jasmonic acid (JA) and salicylic acid (SA) which are important signaling molecules (Theodoulou et al., 2005). Following Pst DC3000, infection the SA levels in both overexpressor lines and pap5-1 plants were $\sim 60 \%$ of the wildtype plants. These results also suggest that $\mathrm{SA}$ accumulation is not completely abolished in both transgenic (35S:PAP5) and pap5-1 plants.

It is well recognized that majority of the eukaryotic proteins undergo reversible phosphorylation via protein kinase (PK) and phosphatase (PP) to control major cellular processes. A large family of protein kinases has been characterized and their function in various cellular processes has been well established (País et al., 2009). However, the physiological role of its counter partner protein phosphates has been poorly understood. Activation of sucrose phosphate synthase (SPS) and nitrate reduction (NR) has been associated with decrease in phosphorylation status of SPE and NR (Huber and Huber, 1996). Interestingly, phosphorylation of Ser158 is sufficientfor inactivation of spinach SPS in vitro (Huber and Huber, 1992). Similarly, PAP5 may be required for complete activation of vital enzymes such as glycolate oxidase in peroxisomes that modulate $\mathrm{H}_{2} \mathrm{O}_{2}$ generation (Figure 6). Since ROS is generated under normal metabolic processes, a highly regulated mechanism must exist to control ROS generation on pathogen recognition.

Several PAPs (SAP1, SAP2, AtPAP17, and AtPAP26) induced under Pi starvation are secreted to the extracellular space to hydrolyze $\mathrm{Pi}$ containing substrates and also exhibit peroxidase

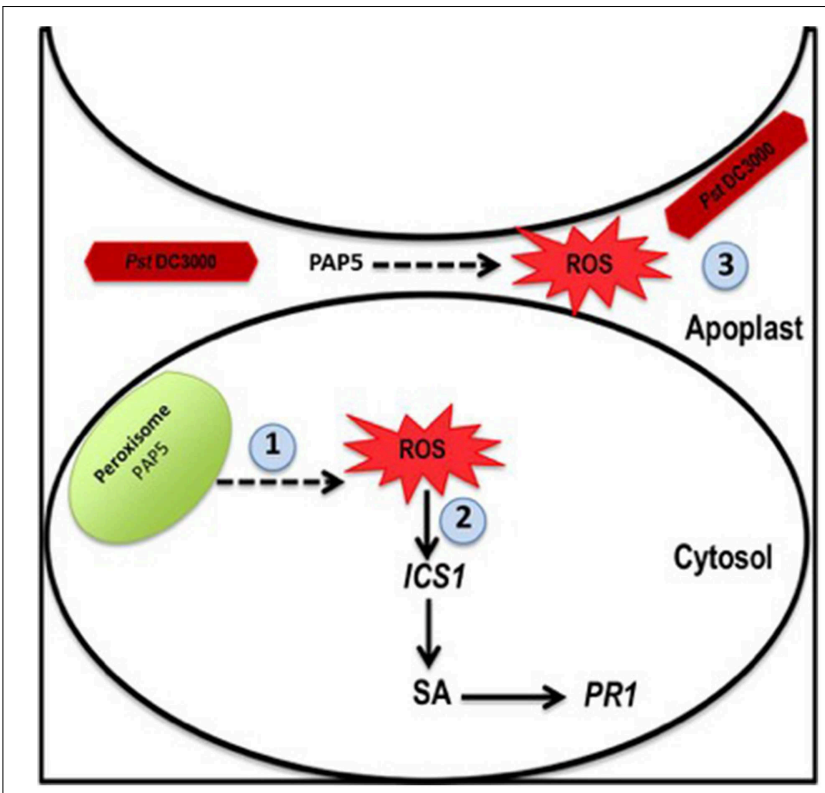

FIGURE 6 | Model for role of PAP5 during Pst DC3000 infection. When plants are infected with virulent Pst DC3000 1. Peroxisomal localized PAP5 may be required for activation of glycolate oxidase, which modulate $\mathrm{H}_{2} \mathrm{O}_{2}$ generation. 2. ROS induces several defense responsive genes including PR1 and ICS1. 3. ROS secreted to the apoplast may directly affect Pst DC3000. Recognition of Pst DC3000, induce expression of PAP5 only during the early stages of infection ( $6 \mathrm{~h}$ ) and triggers ROS synthesis which subsequently activates other defense related signals down stream for complete resistance.

activity (Del Pozo et al., 1999; Bozzo et al., 2002; Hurley et al., 2010). However, the role of PAPs and its peroxidase activity in the extracellular space is not clear. Previously, PAPs exhibiting peroxidase activity has been speculated to function in ROS production similar to the oxidative burst that occurs in response to pathogen recognition (Bozzo et al., 2002; Hurley et al., 2010). Bacterial pathogens including Pst DC3000 reaches the apoplastic region to acquire nutrients (Alfano and Collmer, 1996). It is possible that the PAPs secreted to the extracellular space have dual functions, i.e., the hydrolytic activity under Pi starvation and microbial killing during pathogenesis. Hence, $\mathrm{H}_{2} \mathrm{O}_{2}$ generated in response to Pst DC3000 infection is necessary for activation of basal defense responses. Further, $\mathrm{H}_{2} \mathrm{O}_{2}$ secreted to the extracellular space and apoplast may restrict Pst DC3000 growth directly. Taken together, these evidences suggest that peroxisomal localized PAP5 plays a vital role in basal defense response. Moreover, an optimal level of PAP5 is critical for maintaining complete basal resistance during pathogenesis. It is evident that the isoform of PAP have evolved to attribute various biological functions in plants.

\section{Materials and Methods}

\section{Biological Materials and Growth Conditions}

Arabidopsis thaliana (L.) Heynh, ecotype Columbia (Col-0) seeds were purchased from Lehle seeds (Round Rock, TX, USA) and pap5 T-DNA insertion mutant line was obtained from 
Arabidopsis Biological Resource Center (Columbus, OH, USA). Stratified seeds were planted on Jiffy peat pellets (Halifax seeds, Canada) and seedling were grown at $22 \pm 2^{\circ} \mathrm{C}$ with a photoperiod of $16 \mathrm{~h}$ light at $125 \mu \mathrm{mol} \mathrm{m}{ }^{-2} \mathrm{~s}^{-1}$ and $8 \mathrm{~h}$ dark cycle. Virulent Pseudomonas syringae pv. tomato DC3000 (Pst DC3000) was a kind gift from Dr. Diane Cuppels, Agriculture and Agri Food Canada (AAFC), ON, Canada. Pst DC3000 strains was maintained on King's medium B supplemented with rifampicin $\left(50 \mu \mathrm{g} \mathrm{ml}^{-l}\right)$.

\section{Pathogen Inoculation}

For pathogenicity assay, 4-5 week old plants were spray inoculation with bacterial suspension of virulent Pst DC3000. Plant inoculation and bacterial growth in plant apoplast was determined as described earlier (Ravichandran et al., 2013). In brief, strains of Pst DC3000 was cultured in King's medium B supplemented with rifampicin $\left(50 \mu \mathrm{g} \mathrm{ml}^{-l}\right)$ at $28^{\circ} \mathrm{C}$ until $\mathrm{OD}_{600}$ of 0.8 . Bacterial cells were collected by centrifugation and resuspended in water containing $0.02 \%$ Silwet L-77 (Lehle seeds, USA) to a final concentration of $10^{8}$ c.f.u ml ${ }^{-l}$. Plants were spray inoculated and kept under high humidity for disease development. Leaves were excised (8-10 replicates) from different infected plants and were surface sterilized with ethanol $(75 \% \mathrm{v} / \mathrm{v})$. Four to five samples were made by pooling 2 leaf discs $\left(0.5 \mathrm{~cm}^{2}\right)$ and the samples were ground in sterile water with microfuge tube pestle. The ground tissues were serially diluted and plated on King's B medium containing rifampicin $(50 \mu \mathrm{g} / \mathrm{ml})$. The plates were incubated at $28^{\circ} \mathrm{C}$ and colonies were counter after $48 \mathrm{~h}$. For Pst DC3000 induced gene expression, plants were spray inoculated with bacterial suspension $\left(10^{8}\right.$ c.f.u ml$\left.{ }^{-l}\right)$ and leaf tissues were frozen in liquid nitrogen at the time points indicated.

\section{RNA Extraction and Quantitative Real-time PCR}

Total RNA was extracted from frozen leaf tissues (3 plants per replicate) for two biological replicates using a monophasic extraction method (Chomczynski and Sacchi, 1987). Total RNA was treated with DNase (Promega, WI, USA) and Reverse Transcription was performed with $1 \mu \mathrm{g}$ of total RNA using High-Capacity cDNA Reverse Transcription kit (Applied Biosystems, ON, Canada). Relative transcript levels were assayed by Real-Time PCR using gene specific primers (Table 1) on a StepOnePlus Real-Time PCR system (Applied Biosystems, ON, Canada), using SYBR Green reagent (Applied Biosystems, ON, Canada). To determine relative expression levels, the amount of target gene (three technical replicates/sample) was normalized over the abundance of constitutive GAPDH as endogenous controls. Primers were generated spanning an intron if possible using Primer3Plus (http://www.bioinformatics.nl/cgibin/primer3plus/primer3plus.cgi/).

\section{Quantification of Salicylic Acid (SA) and Hydrogen Peroxide $\left(\mathrm{H}_{2} \mathrm{O}_{2}\right)$}

For SA and JA quantification 4-5 week plants were spray inoculated with Pst DC3000 $\left(10^{8}\right.$ c.f.u ml $\left.{ }^{-l}\right)$. Leaves were excised (five plants per sample) in triplicates at 48 h.p.i and were snap frozen in liquid nitrogen. Leaf tissues $(250 \mathrm{mg})$ were ground with liquid nitrogen and extracted with $5-10 \mathrm{ml}$ of $\mathrm{MeOH}$ $\mathrm{H}_{2} \mathrm{O}-\mathrm{HOAc}$ (90:9:1). After $15 \mathrm{~min}$ the samples were centrifuged
TABLE 1 | List of primer sequences used in RT-qPCR.

\begin{tabular}{|c|c|c|}
\hline Gene & Locus & Primer sequences $\left(5^{\prime}-3^{\prime}\right)$ \\
\hline GAPDH & At1g13440 & $\begin{array}{l}\text { TTGGTGACAACAGGTCAAGCA } \\
\text { AAACTTGTCGCTCAATGCAATC }\end{array}$ \\
\hline ICS1 & At1g74710 & $\begin{array}{l}\text { GCGTCGTTCGGTTACAGG } \\
\text { ACAGCGAGGCTGAATATCAT }\end{array}$ \\
\hline PAP5 & At1g52940 & $\begin{array}{l}\text { AACAGGTCGCTCCACTAGACA } \\
\text { TGGTTAGAGGCATATGTTTGTCC }\end{array}$ \\
\hline PR1 & At2g14610 & $\begin{array}{l}\text { TGATCCTCGTGGGAATTATGT } \\
\text { TGCATGATCACATCATTACTTCAT }\end{array}$ \\
\hline
\end{tabular}

at $12,000 \mathrm{~g}$ for $10 \mathrm{~min}$ and the supernatant was collected. The extraction procedure was repeated twice. The pooled supernatant was dried under steam of $\mathrm{N}_{2}$ and suspended in $5 \mathrm{ml}$ of $0.05 \%$ HOAc in $\mathrm{H}_{2} \mathrm{O}-\mathrm{MeCN}$ (85:15). The samples were then filtered through $0.45 \mu \mathrm{m}$ filter and meanwhile the SampliQ SAX (Aligent technologies, USA) cartridge was conditioned with $2 \mathrm{ml}$ of $\mathrm{MeOH}$. The cartridge was then equilibrated with $5 \mathrm{ml}$ of water. The filtered samples were loaded on to SampliQ SAX cartridge and were washed with $5 \mathrm{ml}$ of $50 \mathrm{mM}$ sodium acetate in $5 \%$ methanol. The interphase (IP) was removed with $5 \mathrm{ml}$ of methanol. SA and JA were eluted with $5 \mathrm{ml}$ of $2 \%$ formic acid in methanol and dried under $\mathrm{N}_{2}$.

After optimization of the liquid chromatography conditions and the tuning of the Orbitrap mass spec for negative mode ionization, a dilution series of salicylic acid (SA) were injected. The detection was performed in negative mode monitoring for the exact mass of the pseudomolecular ion $[\mathrm{M}-\mathrm{H}]^{-}$at $137 \mathrm{amu}$. The samples $(500 \mu \mathrm{g} / \mathrm{ml})$ were analyzed with the same optimized LC/MS method using a Agilent analytical C18 $(3.5 \mu \mathrm{m}, 2.1 \times$ $100 \mathrm{~mm}$ ) with a gradient elution from $5 \% \mathrm{ACN}$ in water to $100 \%$ $\mathrm{ACN}$ using $0.1 \%$ formic acid in both solvents.

For $\mathrm{H}_{2} \mathrm{O}_{2}$ quantification, leaf tissues were harvested (4-5 plants per replicate) for 4 biological replicate and was frozen and ground in liquid nitrogen. To $50 \mathrm{mg}$ of ground frozen tissue, $500 \mu \mathrm{l}$ of phosphate buffer $(50 \mathrm{mM}$, sodium phosphate, $\mathrm{pH}$ 7.4) was added. The samples were centrifuged and $50 \mu \mathrm{l}$ of the aliquot was used for $\mathrm{H}_{2} \mathrm{O}_{2}$ quantification, using an Amplex red hydrogen peroxide/peroxidase assay kit (Molecular Probes, Life Technologies, Canada).

\section{Cloning and Plant Transformation}

The clone of interest was obtained from ABRC and cloned to gateway compatible expression vectors (Earley et al., 2006) using LR Clonase II Gateway Technology (Invitrogen, ON, Canada). Briefly, the clone DQ459170, containing full length PAP5 (Atlg52940) cDNA was obtained from ABRC. The full length cDNA was amplified without the stop codon via polymerase chain reaction (PCR) using TaKaRA Ex Taq ${ }^{\circledR}$ Polymerase (Clontech, USA). The PCR primers were designed to contain attB sites to enable Gateway ${ }^{\circledR}$ technology compatible cloning (Gateway ${ }^{\circledR}$ Technology, Life Technologies, Canada). Shine-Dalgarno and Kozak consensus sequences were included between the attB1 site and the start codon to allow protein expression in E. coli and mammalian cells. The fusion constructs lacking either N/C-terminal of PAP5 was 
generated via PCR. The PAP5 gene lacking N-terminal amino acids MSLETFPPPA (YFP:+30PAP5) was generated using primers $5^{\prime}$-GGTTATAACGCTCCTGAACAAGTT-3' (forward) and $5^{\prime}$-GGTTATAACGCTCCTGAACAAGTT-3' (reverse). PAP5 gene lacking C-terminal amino acids RYYLPEEETI was amplified using primers (-30PAP5:YFP) using primers $5^{\prime}$-ATGTCACTCGAAACATTTCCTC-3' (forward) and $5^{\prime}$ ATTTTTCAACCAAATAGAGTCTGCA-3'. The PCR product was purified and introduced to $\mathrm{pDONR}^{\mathrm{TM}} 221$ vector as per manufactures instruction to generate entry clones (Gateway ${ }^{\circledR}$ Technology, Life Technologies, Canada). The recombinant plasmids were sequenced using the M13 sequencing primers to confirm the insert position and orientation.

The entry clone containing the full length PAP5 or was introduced to the expression vector pEarleyGate 104 (Earley et al., 2006) and pMDC32 (Curtis and Grossniklaus, 2003) using LR clonase (Gateway ${ }^{\circledR}$ Technology, Life Technologies) to generate 35S:YFP-PAP5 and 35S:PAP5 fusion constructs, respectively. Similarly, the fusion constructs lacking either N/Cterminal of PAP5 was introduced to the expression vector pEarleyGate 104. The recombinant plasmids were introduced in to Agrobacterium strain GV310 (pMB90) using the freeze thaw method (Weigel and Glazebrook, 2005). The Agrobacterium strain carrying the fusion construct was used to transform Arabidopsis plants by floral dip method (Clough and Bent, 1998) or infiltrated in to tobacco plants for subcellular localization studies (Sparkes et al., 2006). The floral dip inoculation medium contained 0.5X Murashige and Skoog medium with 5.0\% sucrose and $0.05 \%$ Silwet (Lehle seeds, TX, USA). Plants were selected for Hygromycin and Basta resistance for $\mathrm{pMDC}$ and $\mathrm{pEarley}$ vectors, respectively.

\section{Transient Protein Expression and Subcellular Localization}

For subcellular localization 6 week old Nicotiana benthamiana (tobacco) plants were used. Tobacco plants were grown at 22 $\pm 2^{\circ} \mathrm{C}$ with a photoperiod of $16 \mathrm{~h}$ light at $125 \mu \mathrm{mol} \mathrm{m} \mathrm{m}^{-2} \mathrm{~s}^{-1}$ and $8 \mathrm{~h}$ dark cycle. Plant organelle specific markers were obtained from ABRC (Nelson et al., 2007) and the plasmids were transformed to Agrobacterium strain GV310 (pMB90) using the freeze thaw method (Weigel and Glazebrook, 2005). Agrobacterium strains carrying the recombinant plasmids were

\section{References}

Alfano, J. R., and Collmer, A. (1996). Bacterial pathogens in plants: life up against the wall. Plant Cell 8, 1683-1698. doi: 10.1105/tpc.8.10.1683

Alvarez, M. İ. A. E., Pennell, R. I., Meijer, P.-J., Ishikawa, A., Dixon, R. A., and Lamb, C. (1998). Reactive oxygen intermediates mediate a systemic signal network in the establishment of plant immunity. Cell 92, 773-784. doi: 10.1016/S0092-8674(00)81405-1

Baker, B., Zambryski, P., Staskawicz, B., and Dineshkumar, S. P. (1997). Signaling in plant-microbe interactions. Science 276, 726-733. doi: $10.1126 /$ science. 276.5313 .726

Bari, R., and Jones, J. D. (2009). Role of plant hormones in plant defence responses. Plant Mol. Biol. 69, 473-488. doi: 10.1007/s11103-008-9435-0 grown in liquid Luria-Bertani (LB) media supplemented with appropriate antibiotics. Cells were harvested by centrifugation (5500 $\mathrm{g}$ for $10 \mathrm{~min}$ ) and resuspended in infiltration medium to $\mathrm{OD}_{600}$ of 0.8 . The infiltration medium contained $0.5 \%$ glucose, $50 \mathrm{mM}$ MES, $2 \mathrm{mM} \mathrm{Na} 3 \mathrm{PO}_{4}, 0.1 \mathrm{mM}$ acetosyringone (Sparkes et al., 2006). For co-expression of different constructs Agrobacterium suspension was mixed in equal proportion and the Agrobacterium suspension mixtures were infiltrated to the tobacco leaves using a needleless syringe. The leaf samples were excised $48 \mathrm{~h}$ after infiltration and mounted on a microscope slide in water. The images were obtained (single optical sections and Z-stack) using a Zeiss LSM 510 META inverted confocal laser scanning microscope (Carl Zeiss MicroImaging $\mathrm{GmbH}$ ). For CFP fluorescence, excitation wavelength of $458 \mathrm{~nm}$ was used and emissions were collected between 475, 471 and $525 \mathrm{~nm}$. For YFP fluorescence, an excitation wavelength of $514 \mathrm{~nm}$ was used and emissions were 472 collected between 530 and $600 \mathrm{~nm}$. The fluorescence images were processed using Zeiss LSM Image Browser (Carl Zeiss MicroImaging $\mathrm{GmbH})$.

\section{Acknowledgments}

We would like to thank Dr. Hongxia Liu (Department of Biology, Dalhousie University) for her help and advice with cloning and confocal imaging experiments. We also thank Ms. Margie Tate (Faculty of Agriculture, Dalhousie University) for her assistance with SA quantification. This work was supported by Natural Science and Engineering Research Council of Canada Discovery grant to BP.

\section{Supplementary Material}

The Supplementary Material for this article can be found online at: http://journal.frontiersin.org/article/10.3389/fpls.2015. 00568

Figure S1 | Subcellular localization of PAP5 in N. benthamiana leaves. Agrobacterium strains carrying the recombinant plasmids (35S:YFP-PAP5) and Golgi or plastid specific markers were transiently expressed in tobacco leaves. Left plane shows a single optical section of CFP fluorescence images, middle planes show a single optical section of YFP fluorescence and right planes shows transmitted light images with merged CFP and YFP fluorescence. Bar $=25 \mu \mathrm{m}$. 
tolerance induced by $\mathrm{H}_{2} \mathrm{O}_{2}$ in transgenic tobacco. Proc. Natl. Acad. Sci. U.S.A. 95, 5818-5823. doi: 10.1073/pnas.95.10.5818

Chaouch, S., Queval, G., Vanderauwera, S., Mhamdi, A., Vandorpe, M., LangloisMeurinne, M., et al. (2010). Peroxisomal hydrogen peroxide is coupled to biotic defense responses by ISOCHORISMATE SYNTHASE1 in a daylength-related manner. Plant Physiol. 153, 1692-1705. doi: 10.1104/pp.110.153957

Chen, I. C., Huang, I. C., Liu, M. J., Wang, Z. G., Chung, S. S., and Hsieh, H. L. (2007). Glutathione S-transferase interacting with far-red insensitive 219 is involved in phytochrome A-mediated signaling in Arabidopsis. Plant Physiol. 143, 1189-1202. doi: 10.1104/pp.106.094185

Chisholm, S. T., Coaker, G., Day, B., and Staskawicz, B. J. (2006). Host-microbe interactions: shaping the evolution of the plant immune response. Cell 124, 803-814. doi: 10.1016/j.cell.2006.02.008

Chomczynski, P., and Sacchi, N. (1987). Single-step method of RNA isolation by acid guanidinium thiocyanate-phenol-chloroform extraction. Anal. Biochem. 162, 156-159. doi: 10.1016/0003-2697(87)90021-2

Clough, S. J., and Bent, A. F. (1998). Floral dip: a simplified method forAgrobacterium-mediated transformation of Arabidopsis thaliana. Plant J. 16, 735-743. doi: 10.1046/j.1365-313x.1998.00343.x

Curtis, M. D., and Grossniklaus, U. (2003). A gateway cloning vector set for highthroughput functional analysis of genes in planta. Plant Physiol. 133, 462-469. doi: $10.1104 /$ pp.103.027979

Dangl, J. L., and Jones, J. D. G. (2001). Plant pathogens and integrated defence responses to infection. Nature 411, 826. doi: $10.1038 / 35081161$

Del Pozo, J. C., Allona, I., Rubio, V., Leyva, A., De La Peã A. A., Aragoncillo, C., et al. (1999). A type 5 acid phosphatase gene from Arabidopsis thaliana is induced by phosphate starvation and by some other types of phosphate mobilising/oxidative stress conditions. Plant J. 19, 579-589. doi: 10.1046/j.1365-313X.1999.00562.x

Dixon, R. A. (2001). Natural products and plant disease resistance. Nature 411, 843-847. doi: $10.1038 / 35081178$

Durner, J., Shah, J., and Klessig, D. F. (1997). Salicylic acid and disease resistance in plants. Trends Plant Sci. 2, 266-274. doi: 10.1016/S1360-1385(97)86349-2

Earley, K. W., Haag, J. R., Pontes, O., Opper, K., Juehne, T., Song, K. M., et al. (2006). Gateway-compatible vectors for plant functional genomics and proteomics. Plant J. 45, 616-629. doi: 10.1111/j.1365-313X.2005.02617.x

Foyer, C. H., and Noctor, G. (2003). Redox sensing and signalling associated with reactive oxygen in chloroplasts, peroxisomes and mitochondria. Physiol. Plant. 119, 355-364. doi: 10.1034/j.1399-3054.2003.00223.x

Glazebrook, J. (2005). Contrasting mechanisms of defense against biotrophic and necrotrophic pathogens. Annu. Rev. Phytopathol. 43, 205-227. doi: 10.1146/annurev.phyto.43.040204.135923

Grant, M., and Lamb, C. (2006). Systemic immunity. Curr. Opin. Plant Biol. 9, 414-420. doi: 10.1016/j.pbi.2006.05.013

Huber, S. C., and Huber, J. L. (1992). Role of sucrose-phosphate synthase in sucrose metabolism in leaves. Plant Physiol. 99, 1275-1278. doi: 10.1104/pp.99. 4.1275

Huber, S. C., and Huber, J. L. (1996). Role and regulation of sucrose-phosphate synthase in higher plants. Annu. Rev. Plant Physiol. Plant Mol. Biol. 47, 431-444. doi: 10.1146/annurev.arplant.47.1.431

Hurley, B. A., Tran, H. T., Marty, N. J., Park, J., Snedden, W. A., Mullen, R. T., et al. (2010). The dual-targeted purple acid phosphatase isozyme AtPAP26 is essential for efficient acclimation of Arabidopsis to nutritional phosphate deprivation. Plant Physiol. 153, 1112-1122. doi: 10.1104/pp.110.153270

Jones, J. D., and Dangl, J. L. (2006). The plant immune system. Nature 444, 323-329. doi: $10.1038 /$ nature 05286

Kaida, R., Satoh, Y., Bulone, V., Yamada, Y., Kaku, T., Hayashi, T., et al. (2009). Activation of beta-glucan synthases by wall-bound purple acid phosphatase in tobacco cells. Plant Physiol. 150, 1822-1830. doi: 10.1104/pp.109. 139287

Kaija, H., Alatalo, S. L., Halleen, J. M., Lindqvist, Y., Schneider, G., Kalervo VãÃNãNen, H., et al. (2002). Phosphatase and oxygen radicalgenerating activities of mammalian purple acid phosphatase are functionally independent. Biochem. Biophys. Res. Commun. 292, 128-132. doi: 10.1006/bbrc. 2002.6615

Kliebenstein, D. (2004). Secondary metabolites and plant/environment interactions: a view through Arabidopsis thaliana tinged glasses. Plant Cell Environ. 27, 675-684. doi: 10.1111/j.1365-3040.2004.01180.x
Lamb, C., and Dixon, R. A. (1997). The oxidative burst in plant disease resistance. Annu. Rev. Plant Physiol. Plant Mol. Biol. 48, 251-275. doi: 10.1146/annurev.arplant.48.1.251

Li, D., Zhu, H., Liu, K., Liu, X., Leggewie, G., Udvardi, M., et al. (2002). Purple acid phosphatases of Arabidopsis thaliana. Comparative analysis and differential regulation by phosphate deprivation. J. Biol. Chem. 277, 27772-27781. doi: 10.1074/jbc.M204183200

Liao, H., Wong, F.-L., Phang, T.-H., Cheung, M.-Y., Li, W.-Y. F., Shao, G., et al. (2003). GmPAP3, a novel purple acid phosphatase-like gene in soybean induced by $\mathrm{NaCl}$ stress but not phosphorus deficiency. Gene 318, 103-111. doi: 10.1016/S0378-1119(03)00764-9

Menke, F. L., Van Pelt, J. A., Pieterse, C. M., and Klessig, D. F. (2004) Silencing of the mitogen-activated protein kinase MPK6 compromises disease resistance in Arabidopsis. Plant Cell Online 16, 897-907. doi: 10.1105/tpc. 015552

Mitic, N. N., Smith, S. J., Neves, A., Guddat, L. W., Gahan, L. R., and Schenk, G. (2006). The catalytic mechanisms of binuclear metallohydrolases. Chem. Rev. 106, 3338-3363. doi: 10.1021/cr050318f

Mutka, A. M., Fawley, S., Tsao, T., and Kunkel, B. N. (2013). Auxin promotes susceptibility to Pseudomonas syringae via a mechanism independent of suppression of salicylic acid-mediated defenses. Plant J. 74, 746-754. doi: $10.1111 /$ tpj.12157

Nelson, B. K., Cai, X., and Nebenfuhr, A. (2007). A multicolored set of in vivo organelle markers for co-localization studies in Arabidopsis and other plants. Plant J. 51, 1126-1136. doi: 10.1111/j.1365-313X.2007.03212.x

Nimchuk, Z., Eulgem, T., Holt, B. F. III., and Dangl, J. L. (2003). Recognition and response in the plant immune system. Annu. Rev. Genet. 37, 579-609. doi: 10.1146/annurev.genet.37.110801.142628

Nyathi, Y., and Baker, A. (2006). Plant peroxisomes as a source of signalling molecules. Peroxisomes 1763, 1478-1495. doi: 10.1016/j.bbamcr.2006 08.031

Olczak, M., and Watorek, W. (2003). Two subfamilies of plant purple acid phosphatases. Physiol. Plant. 118, 491-498. doi: 10.1034/j.13993054.2003.00132.x

País, S. M., Téllez-Iñón, M. T., and Capiati, D. A. (2009). Serine/threonine protein phosphatases type 2A and their roles in stress signaling. Plant Signal. Behav. 4, 1013-1015. doi: 10.4161/psb.4.11.9783

Petersen, L. N., Ingle, R. A., Knight, M. R., and Denby, K. J. (2009). OXI1 protein kinase is required for plant immunity against Pseudomonas syringae in Arabidopsis. J. Exp. Bot. 60, 3727-3735. doi: 10.1093/jxb/erp219

Pineiro, M., Gomez-Mena, C., Schaffer, R., Martinez-Zapater, J. M., and Coupland, G. (2003). EARLY BOLTING IN SHORT DAYS is related to chromatin remodeling factors and regulates flowering in Arabidopsis by repressing FT. Plant Cell 15, 1552-1562. doi: 10.1105/tpc.012153

Ravichandran, S., Stone, S., Benkel, B., and Prithiviraj, B. (2013). purple acid phosphatase 5 is required for maintaining basal resistance against Pseudomonas syringae in Arabidopsis. BMC Plant Biol. 13:107. doi: 10.1186/1471-222913-107

Schenk, G., Elliott, T. W., Leung, E., Carrington, L. E., Mitiä, N. A., Gahan, L. R., et al. (2008). Crystal structures of a purple acid phosphatase, representing different steps of this enzyme's catalytic cycle. BMC Struct. Biol. 8:6. doi: $10.1186 / 1472-6807-8-6$

Schenk, G., Guddat, L. W., Ge, Y., Carrington, L. E., Hume, D. A., Hamilton, S., et al. (2000). Identification of mammalian-like purple acid phosphatases in a wide range of plants. Gene 250, 117-125. doi: 10.1016/S0378-1119(00) 00186-4

Schwessinger, B., and Ronald, P. C. (2012). Plant innate immunity: perception of conserved microbial signatures. Annu. Rev. Plant Biol. 63, 451-482. doi: 10.1146/annurev-arplant-042811-105518

Sparkes, I. A., Runions, J., Kearns, A., and Hawes, C. (2006). Rapid, transient expression of fluorescent fusion proteins in tobacco plants and generation of stably transformed plants. Nat. Protoc. 1, 2019-2025. doi: $10.1038 /$ nprot.2006.286

Theodoulou, F. L., Job, K., Slocombe, S. P., Footitt, S., Holdsworth, M., Baker, A., et al. (2005). Jasmonic acid levels are reduced in COMATOSE ATP-binding cassette transporter mutants. Implications for transport of jasmonate precursors into peroxisomes. Plant Physiol. 137, 835-840. doi: $10.1104 /$ pp.105.059352 
Ton, J., Van Pelt, J. A., Van Loon, L. C., and Pieterse, C. M. (2002). Differential effectiveness of salicylate-dependent and jasmonate/ethylenedependent induced resistance in Arabidopsis. Mol. Plant Microbe Interact. 15, 27-34. doi: 10.1094/MPMI.2002.15.1.27

Torres, M. A., Dangl, J. L., and Jones, J. D. G. (2002). Arabidopsis gp91(phox) homologues AtrbohD and AtrbohF are required for accumulation of reactive oxygen intermediates in the plant defense response. Proc. Natl. Acad. Sci. U.S.A. 99, 517-522. doi: 10.1073/pnas.012452499

Uquillas, C., Letelier, I., Blanco, F., Jordana, X., and Holuigue, L. (2004). NPR1-independent activation of immediate early salicylic acid-responsive genes in Arabidopsis. Mol. Plant Microbe Interact. 17, 34-42. doi: 10.1094/MPMI.2004.17.1.34

Vandenabeele, S., Vanderauwera, S., Vuylsteke, M., Rombauts, S., Langebartels, C., Seidlitz, H. K., et al. (2004). Catalase deficiency drastically affects gene expression induced by high light in Arabidopsis thaliana. Plant J. 39, 45-58. doi: 10.1111/j.1365-313X.2004.02105.x

Veljanovski, V., Vanderbeld, B., Knowles, V. L., Snedden, W. A., and Plaxton, W. C. (2006). Biochemical and molecular characterization of AtPAP26, a vacuolar purple acid phosphatase up-regulated in phosphate-deprived Arabidopsis suspension cells and seedlings. Plant Physiol. 142, 1282-1293. doi: 10.1104/pp.106.087171

Vlot, A. C., Dempsey, D. M., Klessig, D. F., and Vlot, A. C. (2009). Salicylic acid, a multifaceted hormone to combat disease. Annu. Rev. Phytopathol. 47, 177-206. doi: 10.1146/annurev.phyto.050908.135202
Weigel, D., and Glazebrook, J. (2005). Transformation of agrobacterium using the freeze-thaw method. CSH Protoc. 2006, 1031-1036. doi: 10.1101/pdb.prot4665

Wildermuth, M. C., Dewdney, J., Wu, G., and Ausubel, F. M. (2002). Isochorismate synthase is required to synthesize salicylic acid for plant defence. Nature 417, 571-571. doi: 10.1038/417571a

Zhang, L., Du, L., Shen, C., Yang, Y., and Poovaiah, B. W. (2014). Regulation of plant immunity through ubiquitin-mediated modulation of Ca2+?calmodulin?AtSR1/CAMTA3 signaling. Plant J. 78, 269-281. doi: $10.1111 /$ tpj. 12473

Zhang, W., Gruszewski, H. A., Chevone, B. I., and Nessler, C. L. (2008). An Arabidopsis purple acid phosphatase with phytase activity increases foliar ascorbate. Plant Physiol. 146, 431-440. doi: 10.1104/pp.107.109934

Conflict of Interest Statement: The authors declare that the research was conducted in the absence of any commercial or financial relationships that could be construed as a potential conflict of interest.

Copyright (๑) 2015 Ravichandran, Stone, Benkel, Zhang, Berrue and Prithiviraj. This is an open-access article distributed under the terms of the Creative Commons Attribution License (CC BY). The use, distribution or reproduction in other forums is permitted, provided the original author(s) or licensor are credited and that the original publication in this journal is cited, in accordance with accepted academic practice. No use, distribution or reproduction is permitted which does not comply with these terms. 